\title{
Health Disorders among Professional Female Hairdressers in Menoufia Governorate, Egypt: A Cross-Sectional Study
}

\author{
${ }^{1}$ Aziza El-Badry, 1, ${ }^{2}$ Manal Al-Batanony and ${ }^{1}$ Faten Younis \\ ${ }^{1}$ Department of Public Health and Community Medicine, Faculty of Medicine, Menoufia \\ University, Menoufia, Egypt. ${ }^{2}$ Unaizah College of Medicine, Qassim University, Kingdom \\ Saudi Arabia.
}

\begin{abstract}
Background: Professional hairdressers are exposed to a variety of chemicals at their workplace. Objective: to assess health disorders among professional female hairdressers in Menoufia governorate, Egypt. Method: this cross sectional study was conducted on 115 female hairdressers in Menoufia governorate with a similar number of non-exposed controls. All participants were subjected to a predesigned questionnaire regarding respiratory, musculoskeletal, and reproductive manifestations followed by spirometry examinations. Data was collected and tabulated. Results: Lower respiratory manifestations including cough, wheezes, dyspnea, and asthma were more among hairdressers $(\mathrm{p}<0.05)$ with highly significant reduction in spirometry measurements $(\mathrm{p}<0.001)$. Musculoskeletal and Reproductive manifestations were significantly higher among hairdressers $(\mathrm{p}<0.05)$ as well. Hairdressers working for more than 9 years were more prone for such disorders. Conclusion: Health and safety of professional hairdressers in Egypt has been inadequately provided as proved by development of respiratory, musculoskeletal and reproductive disorders, which mandates the high need for health education, diagnostic and therapeutic interventions to this work group.
\end{abstract}

Key words: hairdressers, health disorders, spirometry, Respiratory disorders, musculoskeletal. Corresponding author: Aziza El-Badry, m_rezk9207@yahoo.com

\section{Introduction}

Hairdressers are exposed to a variety of harmful agents at their workplace which include chemical agents in hair cosmetic products such as hair dyes, permanent wave solutions and bleaches, physical agents such as noise and temperature and ergonomic hazards due to inappropriate posture during work, and long working hours. $^{1,2}$

Hairdressers are exposed to multiple allergens and irritants during work particularly with a great amount of wet work with subsequent allergic skin and respiratory manifestations. ${ }^{3-6}$

Musculoskeletal disorders have been reported by hairdressers as Neck pain, wrist/hand pain and low back pain. These complaints were related to prolonged sitting, use of vibrating tools, reaching far and awkward body postures. ${ }^{7}$

The aim of this study was to assess health disorders among professional female hairdressers in Menoufia governorate, Egypt.

\section{Method}

This cross-sectional study was conducted at the Department of Public Health and Community Medicine, Menoufia Faculty of Medicine, Menoufia governorate, Egypt, between January 2018 and February, 2019.

Shebin El-Kom city was chosen from Menoufia governorate (as it is the capital 
and the largest city in this governorate). From 29 hair salons in this city, Total number of female hairdressers was 149. After exclusion of non responders and those who had less than one year of employment, the total eligible female hairdressers were 115 with response rate of $90.6 \%$. An equal number of female controls (115 subjects) of office workers from Faculty of Medicine, Menoufia University, never worked as hairdressers.

Women with known respiratory, Gynecological disorders as well as having chronic medical diseases as diabetes mellitus and hypertension were excluded from the study.

A pre-designed questionnaire was filled through personal interviews at the working centers. The questionnaire has four main parts; (1) Personal data including age, residence, educational level and marital status, (2) Occupational data including duration of work in years, working hours per day and work practice data including usage of Personal Protective Equipments (PPEs) as gloves and face masks. (3) Respiratory manifestations including both upper and lower respiratory complaints as running nose, cough, wheezes and difficult breathing. (4) Musculoskeletal manifestations including pain symptoms in the upper \& lower limb, neck and back. (5) Reproductive manifestations including preterm delivery (delivery before completed 37 weeks of gestation) and low birth weight (birth weight less than 2500 gm).

\section{Spirometry measurements:}

All parameters were measured using portable spirometer Spirolab II (Quest medical spirometry and equipment and supplies, MA, USA). The test was done in the sitting position after explanation of the principles of the test. Measurements obtained were expressed as percentage of predicted for standing height, weight, age and sex of tested participants. Forced expiratory maneuvers were repeated until three acceptable tests were obtained and the best forced expiratory volume in the first second (FEV1), forced vital capacity (FVC), and FEV1/ FVC values were recorded.

\section{Statistical analysis}

Data were analyzed using IBM SPSS $®$ ver 22 (SPSS Inc, Chicago, IL, USA).The collected data were summarized as mean \pm SD and range for quantitative data and proportions for qualitative data. Student's $t$ test and Fisher's exact test, were used as appropriate. A $p$ value $<0.05$ was considered statistically significant.

\section{Ethical Consideration}

The study protocol was formally reviewed and approved by the Ethical committee for human research at the Menoufia Faculty of Medicine with informed consent obtained from all participants before starting the study. All measures were in accordance with the declaration of Helsinki as revised in 2000 .

\section{Results}

The work duration (year) of Hairdressers was ranged $0.5-26$ with Mean \pm SD (9.8 \pm 6.9$)$ and median 9. The working hours/day of Hairdressers was ranged $2-$ 16 with Mean \pm SD $(6.8 \pm 2.8)$.

There was no significant difference between the two groups regarding demographic data as depicted in table (1).

Lower respiratory manifestations including cough, wheezes, dyspnea and asthma were more in the study group $(\mathrm{p}<0.05)$ with highly significant difference regarding all respirometric measurements $(\mathrm{p}<0.001)$ as shown in table (2).

Musculoskeletal and Reproductive manifestations were significantly higher among hairdressers $(\mathrm{p}<0.05)$ as revealed in table (3). 
Table (1): Socio-demographic characteristics of studied participants

\begin{tabular}{|c|c|c|c|}
\hline Studied variables & $\begin{array}{l}\text { Hairdressers' group } \\
\text { (No.=115) } \\
\text { N }(\%)\end{array}$ & $\begin{array}{c}\text { Control group } \\
\text { (No.=115) } \\
\text { N }(\%)\end{array}$ & $\begin{array}{c}\mathbf{P} \\
\text { value }\end{array}$ \\
\hline $\begin{array}{l}\text { Age } \\
\text { Mean } \pm \text { SD } \\
\quad \text { Range } \\
\end{array}$ & $\begin{array}{c}30.8 \pm 7.4 \\
19-43 \\
\end{array}$ & $\begin{array}{c}29.7 \pm 7.9 \\
18-49 \\
\end{array}$ & $0.29^{\#}$ \\
\hline $\begin{array}{l}\text { Residence } \\
\text { - Urban } \\
\text { - Rural }\end{array}$ & $\begin{array}{l}72(62.6) \\
43(37.4)\end{array}$ & $\begin{array}{l}63(54.8) \\
52(45.2)\end{array}$ & $0.23^{\# \#}$ \\
\hline $\begin{array}{l}\text { Educational level } \\
\text { - Illiterate and basic } \\
\text { - Secondary and above }\end{array}$ & $\begin{array}{l}81(70.4) \\
34(29.6)\end{array}$ & $\begin{array}{l}74(64.3) \\
41(35.7)\end{array}$ & $0.32^{\# \#}$ \\
\hline $\begin{array}{l}\text { Marital status } \\
\text { - } \quad \text { Single } \\
\text { - } \quad \text { Married } \\
\text { - } \quad \text { Divorced } \\
\text { - } \quad \text { Widow }\end{array}$ & $\begin{array}{r}32(27.8) \\
65(56.5) \\
12(10.4) \\
6(5.2)\end{array}$ & $\begin{array}{r}36(31.3) \\
71(61.7) \\
5(4.3) \\
3(2.6)\end{array}$ & $0.22^{\# \#}$ \\
\hline
\end{tabular}

Table 2: Respiratory Manifestations and Spirometric Measurements of Studied Participants

\begin{tabular}{|c|c|c|c|c|}
\hline Studied variables & $\begin{array}{c}\text { Hairdressers' } \\
\text { Group }(\text { No.=115) } \\
\text { N (\%) }\end{array}$ & $\begin{array}{c}\text { Control group } \\
\text { (No.=115) } \\
\text { N }(\%) \\
\end{array}$ & $P$ value & OR $(95 \% \mathrm{CI})$ \\
\hline Rhin & $18(15.6)$ & 11 (9.6) & $0.16^{\# \#}$ & $1.75(0.79-3.90)$ \\
\hline Eye i & $11(9.6)$ & $6(5.2)$ & $0.21^{\# \#}$ & $1.92(0.69-5.38)$ \\
\hline Cough & $19(16.5)$ & $9(7.8)$ & $\mathbf{0 . 0 4}^{\# \#}$ & $2.33(1.01-5.40)$ \\
\hline Wheezes & $26(22.6)$ & $12(10.4)$ & $\mathbf{0 . 0 1}^{\# \#}$ & $2.51(1.20-5.26)$ \\
\hline Dyspnea & $20(17.4)$ & $10(8.7)$ & $\mathbf{0 . 0 5}^{\# \#}$ & $2.21(1.19-4.96)$ \\
\hline Chronic & $5(4.4)$ & $2(1.7)$ & $0.25^{\# \# \#}$ & $9-13.52)$ \\
\hline Asthma & & $1(0.87)$ & & \\
\hline FVC\% & $\begin{array}{l}\text { Mea } \\
79.4\end{array}$ & \multicolumn{2}{|l|}{ Mean \pm SD } & $\mathbf{0 . 0 0 1}^{\#}$ \\
\hline $\mathrm{FEV}_{1} \%$ & $74.8 \pm 7.4$ & \multicolumn{2}{|l|}{$85.7 \pm 6.9$} & $<\mathbf{0 . 0 0 1}^{\#}$ \\
\hline FEV $_{1} / \mathrm{FVC} \%$ & $80.1 \pm 10.7$ & \multicolumn{2}{|l|}{$\frac{05.1 \pm 0.9}{91.3 \pm 11.3}$} & $\mathbf{0 1}{ }^{\#}$ \\
\hline $\mathrm{FEF}_{25-75} \%$ & $71.6 \pm 14.3$ & \multicolumn{3}{|l|}{$86.3 \pm 12.5$} \\
\hline $\begin{array}{l}{ }^{\#} t \text {-test }{ }^{\# \#} \chi^{2} \text { test }^{\# \# \#} I \\
\text { Regarding the durat } \\
\text { hairdressers working } \\
\text { were more prone for } \\
\text { (table 4) as well as } \\
\text { Reproductive manifes } \\
\text { Discussion } \\
\text { The current study c } \\
\text { affection among prof } \\
\text { which was worse wit } \\
\text { of employment more }\end{array}$ & $\begin{array}{l}\text { of exact } \\
\text { more than } 9 \text { years } \\
\text { spiratory affection } \\
\text { usculoskeletal and } \\
\text { ions (table 5). }\end{array}$ & \multicolumn{3}{|c|}{$\begin{array}{l}\text { than demographic criteria, and these } \\
\text { manifestations were further potentiated by } \\
\text { the spirometry measurements. } \\
\text { Although most of the beauty centers at } \\
\text { Menoufia governorate have mechanical } \\
\text { ventilations as fans and air conditioning } \\
\text { devices, few professional hairdressers } \\
\text { regularly use the personal protective } \\
\text { equipments as gloves and face masks } \\
\text { which could explain the severity of } \\
\text { respiratory complaints during working. }\end{array}$} \\
\hline
\end{tabular}


Table 3: Musculoskeletal and gynecological manifestations of studied participants

\begin{tabular}{|c|c|c|c|c|}
\hline Studied variables & $\begin{array}{c}\text { Hairdressers' group } \\
(\text { No. }=115) \\
\text { N }(\%)\end{array}$ & $\begin{array}{c}\text { Control group } \\
\text { (No.=115) } \\
\text { N }(\%) \\
\end{array}$ & P value & OR $(95 \% \mathrm{CI})$ \\
\hline Neck pain & $32(27.8)$ & $11(9.6)$ & $\mathbf{0 . 0 0 4} 4^{\# \#}$ & $3.65(1.73-7.67)$ \\
\hline Shoulder pain & $37(32.2)$ & $17(14.8)$ & $\mathbf{0 . 0 0 2} 2^{\# \#}$ & $2.73(1.43-5.22)$ \\
\hline Elbow pain & $7(6.1)$ & $2(1.7)$ & $0.09^{\# \# \#}$ & $3.66(0.74-18.02)$ \\
\hline Wrist and hand pain & $29(25.2)$ & $12(10.4)$ & $\mathbf{0 . 0 0 3}^{\# \#}$ & $2.89(1.39-6.01)$ \\
\hline Upper back pain & $27(23.5)$ & $7(6.1)$ & $<0.001^{\# \#}$ & $4.73(1.97-11.39)$ \\
\hline Lower back pain & $42(36.5)$ & $20(17.4)$ & $\mathbf{0 . 0 0 1}^{\# \#}$ & $2.73(1.48-5.05)$ \\
\hline Leg/foot pain & $35(30.4)$ & $11(9.6)$ & $<0.001^{\# \#}$ & $4.14(1.98-8.65)$ \\
\hline $\begin{array}{l}\text { Preterm delivery } \\
<37\end{array}$ & $\begin{array}{r}(\mathrm{n}=83) \\
9(10.8)\end{array}$ & $\begin{array}{l}(\mathrm{n}=79) \\
2(2.5)\end{array}$ & $\mathbf{0 . 0 4} 4^{\# \#}$ & $4.68(1.11-22.40)$ \\
\hline $\begin{array}{l}\text { Low Birth Weight } \\
<2500\end{array}$ & $\begin{array}{l}(\mathrm{n}=83) \\
4(4.8)\end{array}$ & $\begin{array}{r}(\mathrm{n}=79) \\
1(1.3)\end{array}$ & $0.19^{\# \# \#}$ & $3.95(0.43-36.13)$ \\
\hline
\end{tabular}

${ }^{\# \#} \chi^{2}$ test ${ }^{\# \# \#}$ Fisher's exact test

Hairdressing practices of bleaching, dye application and wave application have

been linked with occurrence of allergic symptoms among hairdressers. $5,6,8$

Table 4: Respiratory Manifestations and Spirometry Measurements of Hairdressers Regarding Duration of Employment

\begin{tabular}{|c|c|c|c|c|}
\hline \multirow[t]{2}{*}{ Studied variables } & \multicolumn{2}{|c|}{$\begin{array}{c}\text { Duration of employment } \\
\text { of hairdresser's group } \\
\text { (No.=115) }\end{array}$} & \multirow[t]{2}{*}{$P$ value } & \multirow[t]{2}{*}{ OR $(95 \% \mathrm{CI})$} \\
\hline & $\begin{array}{c}<9 \text { years } \\
(\mathrm{No.}=53) \\
\mathrm{N}(\%)\end{array}$ & $\begin{array}{l}\geq 9 \text { years } \\
(\mathrm{No.}=62) \\
\mathrm{N}(\%)\end{array}$ & & \\
\hline Rhinitis & $8(15.1)$ & $10(16.1)$ & $0.88^{\# \#}$ & $1.08(0.39-2.98)$ \\
\hline Eye irritation & $5(9.4)$ & $6(9.7)$ & $0.96^{\# \#}$ & $1.03(0.30-3.58)$ \\
\hline Cough & $4(7.5)$ & $15(24.2)$ & $\mathbf{0 . 0 2} 2^{\# \#}$ & $3.91(1.21-12.64)$ \\
\hline Wheezes & $6(11.3)$ & $20(32.3)$ & $0.007^{\# \#}$ & $3.73(1.37-10.17)$ \\
\hline Dyspnea & $5(9.4)$ & $15(24.2)$ & $\mathbf{0 . 0 4}^{\# \#}$ & $3.06(1.03-9.10)$ \\
\hline Chronic bronchitis & $0(0.0)$ & $5(8.1)$ & $\mathbf{0 . 0 3}^{\# \# \#}$ & - \\
\hline Asthma & $0(0.0)$ & $7(11.3)$ & $\mathbf{0 . 0 1}{ }^{\# \#}$ & - \\
\hline & Mean \pm SD & Mean \pm SD & & \\
\hline FVC\% & $78.3 \pm 6.2$ & $75.8 \pm 3.9$ & & $\mathbf{0 . 0 1}{ }^{\#}$ \\
\hline $\operatorname{FEV}_{1} \%$ & $75.2 \pm 4.4$ & $72.3 \pm 7.6$ & & $\mathbf{0 . 0 2}{ }^{\#}$ \\
\hline $\mathrm{FEV}_{1} / \mathrm{FVC} \%$ & $82.2 \pm 7.9$ & $78.2 \pm 10.3$ & & $\mathbf{0 . 0 2}{ }^{\#}$ \\
\hline $\mathrm{FEF}_{25-75} \%$ & $73.9 \pm 9.3$ & $70.2 \pm 8.5$ & & $\mathbf{0 . 0 3}^{\#}$ \\
\hline
\end{tabular}

Exposure to volatile solvents, propellants and aerosols in hair sprays inside the hairdressing centers in addition to different hair creams products containing formaldehyde, methacrylate's and nitrosamines, were proven to be incriminated in respiratory and allergic manifestations among hairdressers. ${ }^{9}$
Neck pain, wrist/hand pain and low back pain were commonly reported by the hairdressers in this study. These complaints were related to prolonged sitting, use of vibrating tools, reaching far and awkward body postures as previously reported. ${ }^{7}$

Worsening of symptoms and pulmonary function at workplace, and alleviating the
The Egyptian Journal of Community Medicine
Vol. 38
No. 4
Oct. 
Table 5: Musculoskeletal and Gynecological Manifestations of Hairdressers Regarding Duration of Employment

\begin{tabular}{|c|c|c|c|c|}
\hline \multirow[t]{2}{*}{ Studied variables } & \multicolumn{2}{|c|}{$\begin{array}{c}\text { Duration of employment of } \\
\text { hairdresser's group } \\
\text { (No.=115) }\end{array}$} & \multirow[t]{2}{*}{$P$ value } & \multirow[t]{2}{*}{ OR $(95 \% \mathrm{CI})$} \\
\hline & $\begin{array}{c}<9 \text { years } \\
(\mathrm{No.}=53) \\
\mathrm{N}(\%) \\
\end{array}$ & $\begin{array}{c}\geq 9 \text { years } \\
(\mathrm{No.}=62) \\
\mathrm{N}(\%)\end{array}$ & & \\
\hline Neck pain & $10(18.9)$ & $22(35.5)$ & $\mathbf{0 . 0 4}^{\# \#}$ & $2.37(1.01-5.60)$ \\
\hline Shoulder pain & $12(22.6)$ & $25(40.3)$ & $\mathbf{0 . 0 4} 4^{\# \#}$ & $2.31(1.02-5.24)$ \\
\hline Elbow pain & $1(1.9)$ & $6(9.7)$ & $0.08^{\# \# \#}$ & $5.57(0.65-47.85)$ \\
\hline Wrist and hand pain & $8(15.1)$ & $21(33.9)$ & $\mathbf{0 . 0 2}^{\# \#}$ & $2.88(1.15-7.21)$ \\
\hline Upper back pain & $7(13.2)$ & $20(32.3)$ & $\mathbf{0 . 0 2} 2^{\# \#}$ & $3.13(1.20-8.15)$ \\
\hline Lower back pain & $11(20.8)$ & $31(50.0)$ & $\mathbf{0 . 0 0 1}^{\# \#}$ & $3.82(1.67-8.75)$ \\
\hline Leg/foot pain & $15(28.3)$ & $20(32.3)$ & $0.65^{\# \#}$ & $1.21(0.54-2.69)$ \\
\hline Preterm delivery <37 & $\begin{array}{l}(\mathrm{n}=36) \\
\quad 4(11.1)\end{array}$ & $\begin{array}{l}(\mathrm{n}=47) \\
5(10.6)\end{array}$ & $0.95^{\# \# \#}$ & $0.95(0.24-3.83)$ \\
\hline $\begin{array}{l}\text { Low } \\
<2500\end{array}$ Birth Weight & $1(2.8)$ & $3(6.4)$ & $0.45^{\# \# \#}$ & $2.39(0.24-23.95)$ \\
\hline
\end{tabular}

symptoms at home, indicate that they may be related to occupational exposure as demonstrated in a previous cohort study. ${ }^{10}$ Also, a previous cross-sectional study was conducted on 80 female hairdressers and 50 matched controls in Egypt has demonstrated significant associations between hairdressing and both respiratory and musculoskeletal manifestations which was significantly increased with advancing age and higher body mass index. ${ }^{11}$

In this study, reproductive affection was more prominent regarding preterm delivery with all its complications in the neonate including mortality.

A recent meta-analysis revealed a significantly increased risk of fetal death (OR 1.14, $95 \%$ CI 1.04-1.24), and preterm delivery (OR 1.04, $95 \%$ CI 1.00-1.07) among hairdressers and cosmetologists. ${ }^{12}$

The larger cohort included as well as spirometry evaluation constitute the main strength of this study.

Inability to address other health disorders among hairdressers as infertility with its respective investigations was unintended limitation of the current study.
Future research should focus on the type of educational training considering the perceived benefits and disadvantages particularly regarding ventilation and personal protective equipments, while developing plans to decrease health hazards among professional hairdressers.

\section{Conclusion}

Health and safety of professional hairdressers in Egypt has been inadequately provided as proved by development of respiratory, musculoskeletal and reproductive disorders; which mandates the high need for health education, diagnostic and therapeutic interventions to this work group.

\section{Acknowledgements}

We would like to thank the responders for their time to provide valuable information.

Conflicts of Interest: None declared

\section{References:}

1. Hollund, B. E., Moen, B. E., Lygre, S. H., Florvaag, E., \& Omenaas, E. Prevalence of airway symptoms among hairdressers in 
Bergen, Norway. Occupational and Environmental Medicine 2001; 58(12):780-5.

2. Mussi, G., \& Gouveia, N. Prevalence of work-related musculoskeletal disorders in Brazilian hairdressers. Occupational Medicine (London) 2008;52:645-651.

3. Figueiredo, J. P., Pomiecinski, F., Yang, A. C., Castro, F. F., Kalil, J., \& Galvao, C. E. Diagnostic assessment of occupational asthma due to persulfate salts in a professional hairdresser: A case report. Clinics (Sao Paulo),2008; 63:149-150.

4. Gala, O. G., Gancedo, S. Q., Ordóñez, R. F., Camo, I. P., Mancebo, E. G., Agustín, M. C., \& Cosmes, E. L. Diagnostic approach and management of occupational asthma by persulfate salts in a hairdresser. Allergy and Asthma Proceedings 2001; 22: 235-238.

5. Leino, T., Tammilehto, L., Luukkonen, R., \& Nordman, H. (1997). Self reported respiratory symptoms and diseases among hairdressers. Occupational and Environmental Medicine 1997;54: 452-455.

6. Macchioni, P., Kotopulos, C., Talini, D., De Santis, M., Masino, E., \& Paggiaro, P. L. Asthma in hairdressers: A report of 5 cases. Medicina del Lavoro, 1999; 90:776-785.
7. Tsigonia, A., Tanagra, D., Linos, A., Merekoulias, G., \& Alexopoulos, E. C. Musculoskeletal disorders among cosmetologists. International Journal of Environmental Research and Public Health,2009; 6: 2967-2979.

8. Schwartz, H. J. Effect of chronic chromolyn sodium therapy in a beautician with occupational asthma. Journal of Occupational Medicine 1989; 31:112-114.

9. Takkouche, B., Regueira-Méndez, C., \& Montes-Martínez, A. Risk of cancer among hairdressers and related workers: A metaanalysis. International Journal of Epidemiology 2009;38:1512-1531.

10. Skoufi GI, Nena E, Kostikas K, et al. Work-related respiratory symptoms and airway disease in hairdressers. Int $\mathbf{J}$ Occup Environ Med 2013;4:53-60.

11. Hassan OM, Bayomy H. Occupational Respiratory and Musculoskeletal Symptoms among Egyptian Female Hairdressers. J Community Health. 2015;40(4):670-9.

12. Kim D, Kang MY, Choi S, Park J, Lee HJ, Kim EA. Reproductive disorders among cosmetologists and hairdressers: a metaanalysis. Int Arch Occup Environ Health. 2016;89(5):739-53. 